\title{
Non-invasive vibrational mode spectroscopy of ion Coulomb crystals through resonant collective coupling to an optical cavity field
}

\author{
A. Dantan, J. P. Marler, M. Albert, D. Guénot and M. Drewsen* \\ QUANTOP, Danish National Research Foundation Center for Quantum Optics, \\ Department of Physics and Astronomy, University of Aarhus, DK-8000 Arhus C., Denmark
}

(Dated: November 11, 2018)

\begin{abstract}
We report on a novel non-invasive method to determine the normal mode frequencies of ion Coulomb crystals in traps based on the resonance enhanced collective coupling between the electronic states of the ions and an optical cavity field at the single photon level. Excitations of the normal modes are observed through a Doppler broadening of the resonance. An excellent agreement with the predictions of a zero-temperature uniformly charged liquid plasma model is found. The technique opens up for investigations of the heating and damping of cold plasma modes, as well as the coupling between them.

PACS numbers: 37.30.+i,52.27.Gr,37.10.Vz,52.27.Jt,42.50.Pq
\end{abstract}

Cold one-component plasma physics [1] has in the past two decades led to a series of interesting results due to the availability of fast computers [2 [5] as well as the possibility to experiment with ensembles of trapped, lasercooled atomic ions [6 16]. Prominent examples are the understanding of structural properties of crystallized cold plasmas in both Penning [6, 7] and Paul [8 10, 15, 16 traps, and the investigation of the normal mode dynamics of cold magnetized plasmas in Penning traps [11 14]. While there exist many similarities between experiments in Penning and Paul traps, the unmagnetized plasmas in Paul traps are e.g. known to heat up much faster than the magnetized plasmas in Penning traps due to the presence of the rf fields [3, 17]. The lack of a rotational symmetry axis in Paul traps has as well been found to be responsible for the observation of specific crystalline structures [16]. Exploration of the normal mode dynamics of unmagnetized ion Coulomb crystals in e.g. linear Paul traps will hence add to our understanding of the influence of the trapping environment on the physics of such crystals. Furthermore, since large Coulomb crystals are excellent candidates for the realization of high-fidelity quantum memories for light [18], such studies can shed light on the influence of the excitation of these modes on the fidelity as well as on the prospect of storing several photonic quantum bits through deliberate excitations of specific vibrational modes. Larger Coulomb crystals have as well recently been considered as a system for performing quantum simulations, in which respect knowledge of normal mode dynamics is needed [19]. Finally, ion Coulomb crystals represent extremely interesting systems to study cavity optomechanics phenomena [20, 21], since, in spite of their solid nature, they possess free atomic resonance properties and can hence be made very sensitive to the radiation pressure force exerted by optical fields.

In this Letter, we report on the study of normal mode vibrations of Coulomb crystals of ${ }^{40} \mathrm{Ca}^{+}$ions in a linear Paul trap by a novel non-invasive technique. The technique is based on monitoring the coherent collec- tive resonant response of the atomic ions constituting the crystal to a single photon optical cavity field [18]. By having a standing wave optical cavity incorporated in the trap setup with the light propagation axis coinciding with the rf-field free axis of the trap, we can detect very small changes in the optical response due to Doppler shifts resulting from the ions' motion along the cavity axis when a mode is excited. Since the probing does not rely on the observation of incoherently scattered photons as e.g. the case in Doppler velocimetry [11 13] and in Sympathetically-Cooled Single Ion Mass Spectrometry 22], the detected signal can in principle be purely dispersive in nature and does not require any excitation of the ions. The technique is not limited to linear Paul traps, but should work as well in any open structured trap geometries such as e.g. the Penning-Malmberg type traps [11, 23].

Previous Doppler velocimetry imaging experiments with cold magnetized plasmas of $\mathrm{Be}^{+}$ions in a Penning trap have led to the identification of a series of normal modes [11 13], which correlate to specific $(l, m)$-modes theoretically predicted for spheroidal shaped uniformly charged liquids 24]. For the cold unmagnetized plasmas in the linear Paul trap used in our investigations, the corresponding low-order normal mode frequencies are expected to be close to those of the $(l, m)$ modes, which are given by 24]

$$
\omega_{(l, m)}=\frac{\omega_{p}}{\sqrt{1-\frac{P_{l}^{m} Q_{l}^{m^{\prime}}}{Q_{l}^{m} P_{l}^{m^{\prime}}}}},
$$

where $\omega_{p}=\sqrt{q^{2} n_{0} / M \varepsilon_{0}}$ is the plasma frequency (q is the charge of the ion, $\mathrm{M}$ its mass and $n_{0}$ the uniform ion density), $P_{l}^{m}=P_{l}^{m}\left(1 / \sqrt{1-\alpha^{2}}\right)$ and $Q_{l}^{m}=Q_{l}^{m}\left(1 / \sqrt{1-\alpha^{2}}\right)$ are first and second-order Legendre polynomials with cylindrical indices $(l, m)$ and with the prime denoting differentiation with respect to the entire argument. Finally, $\alpha$ is the aspect ratio of the plasma given by $\alpha=R / L$, where $L$ and $R$ are the half-length and radius of the 


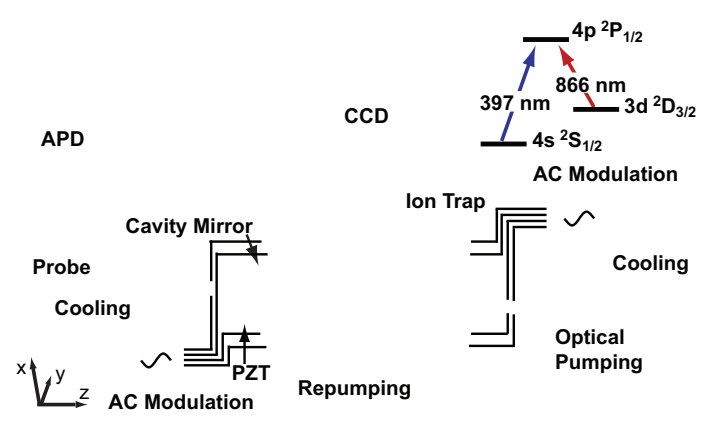

FIG. 1: (color online.) Schematic of the linear Paul trap with an integrated optical cavity. The excitation of the crystal modes (AC modulation) can be monitored either by measuring the change in the reflection spectrum of a weak cavity field $(866 \mathrm{~nm})$ with an avalanche photodiode (APD) or by imaging the fluorescent light $(397 \mathrm{~nm})$ emitted by the ${ }^{40} \mathrm{Ca}^{+}$ions during laser cooling onto a CCD camera. The inset shows the relevant energy levels and transitions of the ${ }^{40} \mathrm{Ca}^{+}$ion.

spheroid, respectively (see also Fig. 2a).

The corresponding spatial structure of the modes can generally be expressed in terms of generalized spheroidal coordinates [25]. While the modes generally have a nontrivial dependence on the ions' position in the crystal, for longitudinal modes $(l, m=0)$, the displacement $\delta z$, close $(r \simeq 0)$ to the axis of rotational symmetry ( $z$-axis), is approximately given by

$$
\delta z \propto P_{l}^{0^{\prime}}\left(z / \sqrt{L^{2}-R^{2}}\right) .
$$

Consequently, all modes with $m=0$ have a spatial variation along the $z$-axis which, under excitation, will lead to measurable Doppler-shifts of the ions resonance frequency along this axis.

Figure 1 shows a schematic of the experimental setup for our studies (a more detailed description of the cavitytrap setup can be found in Ref. [26]). The linear Paul trap consists of four cylindrical electrodes to which an rf voltage $U_{r f}$ between $200 \mathrm{~V}$ and $300 \mathrm{~V}$ is applied at a frequency $\Omega$ of $2 \pi \times 4 \mathrm{MHz}$ for radial confinement ( $x y$ plane in Fig. (1). To obtain a linear quadrupole configuration, the rf voltages are $180^{\circ}$ out of phase between nearest neighbor electrodes. Each of the four electrodes are sectioned into three parts to allow for the application of DC (1-10 V) and AC voltages to the eight end-pieces for static confinement of the ions along the cavity axis ( $z$-axis) and for normal mode excitations, respectively. The electrodes have diameters of $5.2 \mathrm{~mm}$ and the nearest neighbour electrode spacing is $1.8 \mathrm{~mm}$. With these parameters, the single ion effective (pseudo) potential is to a very good approximation harmonic in all dimensions in the region of the crystals, which leads to constant densities of the crystallized ${ }^{40} \mathrm{Ca}^{+}$ions with values between $2.6 \times 10^{8}$ and $5.7 \times 10^{8} \mathrm{~cm}^{-3}$ [27].

The ${ }^{40} \mathrm{Ca}^{+}$ions are produced through isotope selective photoionization of atoms in a beam of natural abun- dant calcium [26, 28]. The Coulomb crystals are created through Doppler laser-cooling along the trap axis by two counter-propagating beams at $397 \mathrm{~nm}$ (total power of $\sim 4 \mathrm{~mW}$ and beam diameter of $\sim 1 \mathrm{~mm}$ ) and an 866 $\mathrm{nm}$ beam applied from the side to prevent the ions from being shelved into the $\mathrm{D}_{3 / 2}$ state (see insert of Fig. 1). The number of ions constituting the crystals can be deduced by imaging the light $(397 \mathrm{~nm})$ scattered by the ions during the cooling process onto a charge coupled device (CCD) chip [26].

Normal modes of the ion Coulomb crystals can be excited by applying identical AC electrical potentials at variable frequency to the four end-pieces at each end of the trap. By this simple geometry modes corresponding to the $(l, m=0)$ charged liquid modes can easily be excited by having the voltages applied at each end either in-phase ( $l$ even) or $180^{\circ}$ out of phase $(l$ odd).

To monitor the coherent collective resonant response of the atomic ions constituting the crystal, a $11.8 \mathrm{~mm}$ long optical cavity with a measured $\mathrm{TEM}_{00}$ mode waist of $37 \mu \mathrm{m}$ is used [26]. The optical finesse of the cavity is about 3,000 at the $866 \mathrm{~nm}$ resonant wavelength of the $3 \mathrm{~d}^{2} \mathrm{D}_{3 / 2}-4 \mathrm{p}{ }^{2} \mathrm{P}_{1 / 2}$ transition in the ${ }^{40} \mathrm{Ca}^{+}$ion addressed in the experiments.

The plasma mode diagnostic involves a sequence of steps. First, a Doppler cooling period of $5 \mu \mathrm{s}$ is followed by a period of $12 \mu \mathrm{s}$, where the ions in the crystal are prepared in the $m_{J}=+3 / 2$ magnetic sub-state of the long-lived metastable $\mathrm{D}_{3 / 2}$ level by optical pumping (efficiency $\sim 97 \%$ ). Next, a weak left-handed circularlypolarized pulse (1.4 $\mu$ s long) of $866 \mathrm{~nm}$ light is coupled into the cavity to probe the collective response of the ions. The mean intracavity photon number is less or about one at any time. During this probing period, the photons reflected by the cavity are measured by an avalanche photodiode with an overall collection efficiency of $16 \%$. This sequence is repeated at a rate of $50 \mathrm{kHz}$ while the cavity length is scanned at a rate of $30 \mathrm{~Hz}$. The cavity lineshape is reconstructed by averaging a few hundred scans (see [18] for more details).

With the ion Coulomb crystal at rest, the cavity probe linewidth is given by [18]:

$$
\kappa^{\prime}=\kappa+g_{N}^{2} \frac{\gamma}{\left(\gamma^{2}+\Delta^{2}\right)},
$$

where $\kappa$ is the cavity decay rate, $g_{N}$ the collective coupling rate, $\gamma$ the optical dipole decay rate and $\Delta$ the detuning of the probe with respect to atomic resonance. If the crystal is moving with a fixed velocity $v_{z}$ along the cavity axis the probe linewidth would due to the Doppler shift be given by

$$
\kappa^{\prime}=\kappa+g_{N}^{2} \frac{\gamma\left(\gamma^{2}+\Delta^{2}+\left(k v_{z}\right)^{2}\right)}{\left(\gamma^{2}+\Delta^{2}\right)^{2}+2\left(\gamma^{2}-\Delta^{2}\right)\left(k v_{z}\right)^{2}+\left(k v_{z}\right)^{4}}
$$

where $k$ is the wavevector of the cavity field. 


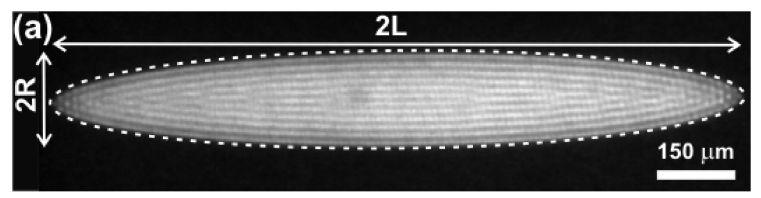

(b)

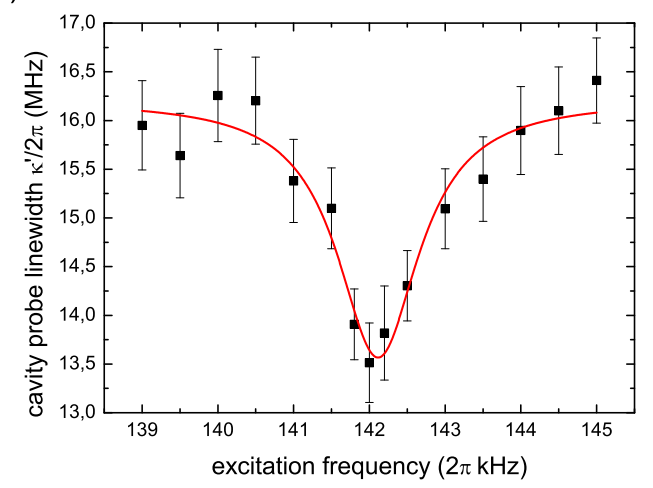

FIG. 2: (a) Projection image of a $1.2 \mathrm{~mm}$-long crystal with 4000 ions and density $2.6 \times 10^{8} \mathrm{~cm}^{-3}$. The DC and rf voltage amplitudes are $2.36 \mathrm{~V}$ and $205 \mathrm{~V}$, respectively, and the aspect ratio $\alpha=R / L$ is 0.135 . (b) Cavity probe linewidth as a function of the mode excitation frequency applied to drive the equivalent of the $(2,0)$ mode of this crystal. The probe is tuned to atomic resonance $(\Delta=0)$ and the $\mathrm{AC}$ modulation voltage is $0.75 \mathrm{~V}$. The red line is a Lorentzian fit.

When exciting one of the normal modes, all the ions in the crystal will generally not have the same velocity at a given instance (this is only true for the center-ofmass mode, see Eq. (2)), and, furthermore, the velocity of the individual ions will change during the mode-period. However, if the timescale at which the velocity of the individual ions changes is slow as compared to the effective cavity photon decay time, one can model the expected probe lineshape by averaging the contributions from the different parts of the crystal over a single mode-oscillation period. From Eqs. (3) and (4) it follows that any excitation of the ions' motion will generally lead to a narrower linewidth due to the reduced coupling strength.

Fig. $2 \mathrm{~b}$ shows the cavity probe linewidth for $\Delta=0$ as a function of the modulation frequency applied to excite the equivalent of the $(2,0)$ "quadrupole" mode of a $1.2 \mathrm{~mm}$-long crystal with 4000 ions at a density $2.6 \times 10^{8} \mathrm{~cm}^{-3}$ (Fig. 2a). A clearly reduced linewidth is observed around $142 \mathrm{kHz}$. The exact resonance value of $\omega_{(2,0)}^{\text {meas }} / 2 \pi=142.1 \pm 0.1 \mathrm{kHz}$ is in good agreement with the resonance frequency $\omega_{(2,0)}^{\text {model }} / 2 \pi=142.2 \pm 1.1 \mathrm{kHz}$ expected for the $(2,0)$ mode of a charged liquid crystal for this aspect ratio and charge density. In order to test more generally how well the uniformly charged liquid model describes unmagnetized ion plasmas confined in a linear Paul trap, the resonance frequencies of the lowest lying normal modes of ion crystals with various aspect

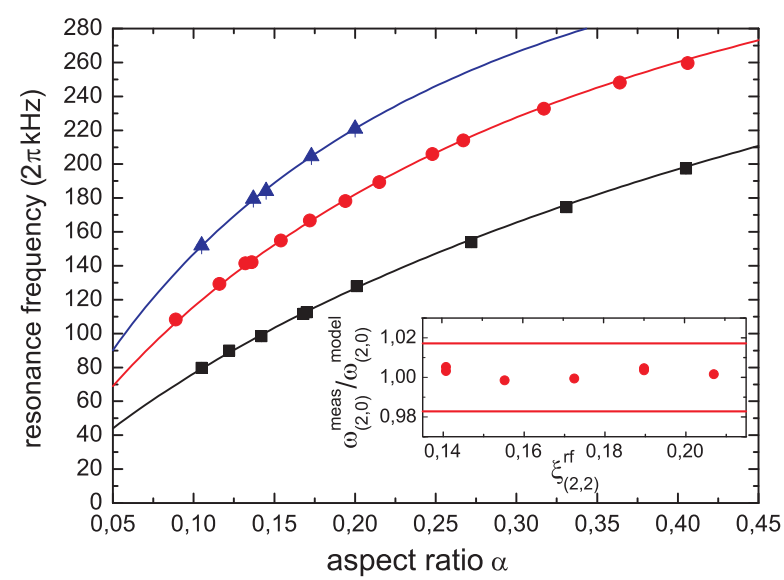

FIG. 3: Resonance frequencies corresponding to the $(1,0)$ (squares), the $(2,0)$ (circles) and the $(3,0)$ (triangles) modes as a function of the aspect ratio $\alpha$, for a fixed plasma frequency $\omega_{p} / 2 \pi=536 \mathrm{kHz}\left(U_{r f}=205 \mathrm{~V}\right)$. The solid lines show the theoretical predictions of Eq. (1). The error bars are within the point size. The inset shows the $(2,0)$ mode resonance frequency as a function of the $(2,2)$ mode off-resonant modulation depth $\xi_{(2,2)}^{r f}$ (see text) for a fixed aspect ratio $\alpha=0.135$. The mode resonance frequency is normalized to that expected for a plasma without any excitation of the $(2,2)$ mode and the red lines show the uncertainty in the expected resonance frequency due to the density calibration. The Coulomb crystals contain between 4000 and 12000 ions, with densities of $2.6-5.6 \times 10^{8} \mathrm{~cm}^{-3}$ and their temperature is in the $10 \mathrm{mK}$ range.

ratios have been determined by monitoring the linewidth of the cavity probe field when tuned to the atomic resonance. The resulting mode resonance frequencies are presented in Fig. 3 together with the predicted values from the charged liquid model (see Eq. (10). The measured values are consistent with the theory to better than one percent for all experimental data. This accuracy may appear quite surprising considering that during all these $(l, 0)$ mode measurements, the $(2,2)$ mode is continuously off-resonantly excited by the linear rf quadrupole field confining the plasma. The rf-induced modulation depth is indeed up up to $20 \%$ of the radial extension of the crystal, i.e. comparable to that typically used for the axial excitation of the $(l, 0)$ modes. The inset of Fig. 3 clearly shows, however, that, within the current experimental accuracy, the $(2,0)$ mode frequency does not have any systematic dependence on the $(2,2)$ mode modulation depth $\xi_{(2,2)}^{r f}$, defined by the micromotion amplitude of an ion at the radial position $\left(x_{0}, y_{0}\right)$ $(x(t), y(t))=\left(x_{0}\left(1+\xi_{(2,2)}^{r f} \cos \Omega t\right), y_{0}\left(1-\xi_{(2,2)}^{r f} \cos \Omega t\right)\right)$. This result is though consistent with molecular dynamics simulations from which it has been predicted that the radial rf field-driven micromotion in linear Paul traps should have an extremely weak coupling into the axial motion of the ions [3]. 


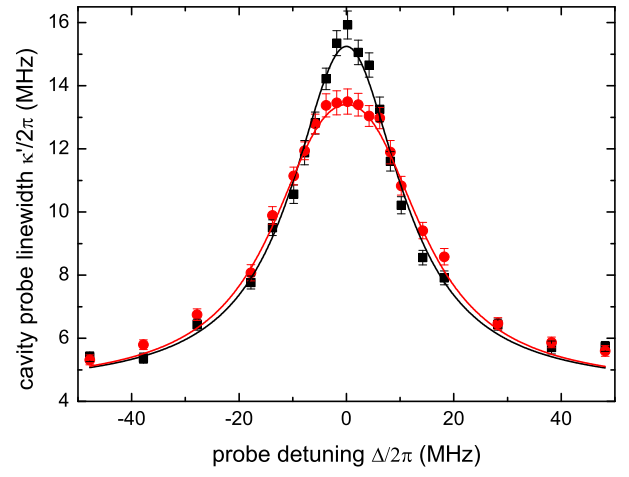

FIG. 4: Cavity probe linewidth as a function of the probe detuning, with (circles) or without (squares) modulation at the $(1,0)$ mode resonance frequency, for a crystal similar to the one presented in Fig. 2 The solid lines are fits according to the theoretical model described in the text.

In Fig. 4. the cavity probe linewidth with and without exciting the $(1,0)$ mode at the resonance frequency $\omega_{(1,0)} / 2 \pi=94 \mathrm{kHz}$ is presented as a function of the detuning of the probe with respect to the atomic resonance. From the Lorentzian line profile in absence of the mode excitation, one finds $\left(\kappa, \gamma, g_{N}\right) / 2 \pi=(2.2 \pm$ $0.1,12.3 \pm 0.3,8.2 \pm 0.1) \mathrm{MHz}$. When the $(1,0)$ mode is excited, the absorption line is substantially modified to a non-Lorenzian profile. A fit based on the $(1,0)$ mode function (Eq. (4) averaged over one oscillation period results in a driven motion amplitude $v_{z 0}^{f i t}=5.9 \pm 0.7$ $\mathrm{m} / \mathrm{s}\left(v_{z}(t)=v_{z 0} \cos \left(\omega_{(1,0)} t+\varphi\right)\right)$. This value is in good agreement with the value $v_{z 0}^{\text {fluo }}=5.3 \pm 0.6 \mathrm{~m} / \mathrm{s}$ deduced from fluorescence images recorded in phase with the modulation voltage. Here, a motional amplitude $z_{0}^{\text {fluo }}=9 \pm 1 \mu \mathrm{m}$ was measured at the mode resonance frequency $\omega_{(1,0)} / 2 \pi=94 \mathrm{kHz}$. Since equally good quantitative agreement between experiments and the model was also found for the $(2,0)$ mode, this proves that quantitative information about the ions' motion can reliably be obtained from the ion-cavity coupling without the need for observing directly the fluorescence signal. In the future, higher order $(l, 0)$ modes are envisioned to be studied through mode excitations using spatial- and timemodulated radiation pressure forces. For ${ }^{40} \mathrm{Ca}^{+}$ions this can e.g. be achieved through the combined application of a $866 \mathrm{~nm}$ repumper beam with a spatially modulated intensity profile along the cavity axis and a time-varying intensity of one of the $397 \mathrm{~nm}$ cooling beams [22]. Eventually, for the high spatial modulation of modes with large $l$, the liquid model should cease to apply. Further applications could be measurements of ion Coulomb crystal temperatures and heating rates [14] and more detailed investigations of the coupling between the various normal modes at various temperatures and structural phases of the plasma [2, 25]. Finally, we also believe that the spectroscopic findings as well as the non-invasive character of the method used will be important for e.g. observing radiation pressure-induced optomechanical effects [20, 21] with ion Coulomb crystals.

We acknowledge financial support from the Carlsberg Foundation and the Danish Natural Science Research Council through the ESF EuroQUAM project CMMC.

* Electronic address: drewsen@phys.au.dk

[1] D. H. E. Dubin and T. M. O’Neil, Rev. Mod. Phys. 71, 87 (1999).

[2] D. H. E. Dubin and J. P. Schiffer, Phys. Rev. E 53, 5249 (1996).

[3] J. P. Schiffer, M. Drewsen, J. F. Hangst, and L. Hornekær, Proc. Natl. Acad. Sci. USA 97, 10697 (2000).

[4] H. Totsuji, T. Kishimoto, C. Totsuji, and K. Tsuruta, Phys. Rev. Lett. 88, 125002 (2002).

[5] T. Matthey, J. P. Hansen, and M. Drewsen, Phys. Rev. Lett. 91, 165001 (2003).

[6] W. M. Itano, J. J. Bollinger, J. N. Tan, B. Jelenković, X.P. Huang, and D. J. Wineland, Science 279, 686 (1998).

[7] T. B. Mitchell, J. J. Bollinger, D. H. E. Dubin, X.-P. Huang, W. M. Itano, and D. J. Wineland, Science 282, 1290 (1998).

[8] D. J. Wineland, J. C. Bergquist, W. M. Itano, J. J. Bollinger, and C. H. Manney, Phys. Rev. Lett. 59, 2935 (1987).

[9] G. Birkl, S. Kassner, and H. Walther, Nature 357, 310 (1992).

[10] M. Drewsen, C. Brodersen, L. Hornekær, J. S. Hangst, and J. P. Schiffer, Phys. Rev. Lett. 81, 2878 (1998).

[11] D. J. Heinzen, J. J. Bollinger, F. L. Moore, W. M. Itano, and D. J. Wineland, Phys. Rev. Lett. 66, 2080 (1991).

[12] J. J. Bollinger, D. J. Heinzen, F. L. Moore, W. M. Itano, D. J. Wineland, and D. H. E. Dubin, Phys. Rev. A 48, 525 (1993).

[13] T. B. Mitchell, J. J. Bollinger, X.-P. Huang, and W. M. Itano, Optics Express 2, 314 (1998).

[14] M. J. Jensen, T. Hasegawa, J. J. Bollinger, and D. H. E. Dubin, Phys. Rev. Lett. 94, 025001 (2005).

[15] A. Mortensen, E. Nielsen, T. Matthey, and M. Drewsen, Phys. Rev. Lett. 96, 103001 (2006).

[16] A. Mortensen, E. Nielsen, T. Matthey, and M. Drewsen, J. Phys. B 40, F223 (2007).

[17] R. Blümel, C. Kappler, W. Quint, and H. Walther, Phys. Rev. A 40, 808 (1989).

[18] P. F. Herskind, A. Dantan, J. P. Marler, M. Albert, and M. Drewsen, Nature Phys. 5, 494 (2009).

[19] D. Porras and J. I. Cirac, Phys. Rev. Lett. 92, 207901 (2004); ibid. 96, 250501 (2006).

[20] K. W. Murch, K. L. Moore, S. Gupta, and D. M. Stamper-Kurn, Nature Phys. 4, 561 (2008).

[21] F. Brenneke, S. Ritter, T. Donner, and T. Esslinger, Science 322, 235 (2008).

[22] M. Drewsen, A. Mortensen, R. Martinussen, P. Staanum, and J. L. Sørensen, Phys. Rev. Lett. 93, 243201 (2004).

[23] C. F. Driscoll, J. H. Malmberg, and K. S. Fine, Phys. 
Rev. Lett. 60, 1290 (1988).

[24] D. H. E. Dubin, Phys. Rev. Lett. 66, 2076 (1991).

[25] D. H. E. Dubin, Phys. Rev. E 53, 5268 (1996).

[26] P. F. Herskind, A. Dantan, M. B. Langkilde-Lauesen, A. Mortensen, J. L. Sørensen, and M. Drewsen, Appl. Phys. B 93, 373 (2008).
[27] P. F. Herskind, A. Dantan, M. Albert, J. P. Marler, and M. Drewsen, J. Phys. B 42, 154008 (2009).

[28] A. Mortensen, J. Lindballe, I. S. Jensen, P. Staanum, D. Voigt, and M. Drewsen, Phys. Rev. A 69, 042502 (2004). 